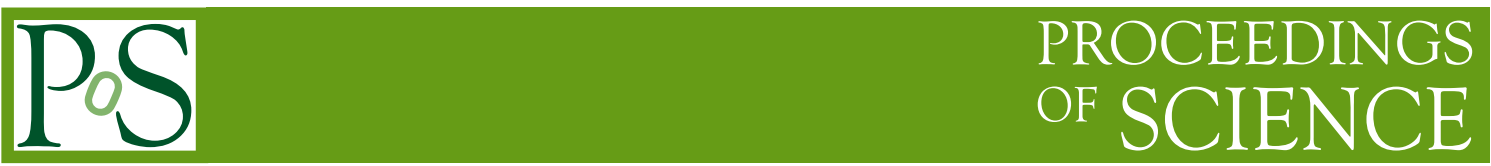

\title{
CP Violation and Leptogenesis
}

\author{
Silvia Pascoli* \\ IPPP, Department of Physics, Durham University, South Road, Durham DH1 3LE, United \\ Kingdom \\ E-mail: silvia.pascoli@durham.ac.uk
}

The origin of the baryon asymmetry of the Universe is one of the key open questions to be addressed in particle physics and cosmology. Assuming a negligible initial baryon asymmetry, its dynamical production can happen in presence of non-conservation of the baryon and/or lepton number, violation of the $\mathrm{C}$ and $\mathrm{CP}$ symmetries and out-of-equilibrium conditions. Leptogenesis is a mechanism, proposed in the context of see-saw models, which can satisfy all these requirements and explain the observed baryon asymmetry. In the simplest case of see-saw type I, in which right handed neutrinos, singlets of the Standard Model, are introduced, lepton number is violated by the heavy Majorana masses, in conjuction with the Yukawa couplings, CP can be violated by complex terms in the Dirac masses and the out-of equilibrium condition is satisfied by the decays of the right handed neutrinos, when the temperature drops below their mass. The interesting question arises if the observable low energy CP violation, parameterised by the $\delta$ phase and measurable in long baseline neutrino oscillation experiments, can be responsible for the baryon asymmetry. Although in a model independent way it is not possible to draw such connection, we show that, in presence of flavour effects in leptogenesis, the $\delta$ phase enters directly in the lepton, and baryon, asymmetry and thanks to the large value of $\theta_{13}$ it may be at the origin of the matter-antimatter asymmetry we observe.

XVI International Workshop on Neutrino Telescopes,

2-6 March 2015

Palazzo Franchetti, Istituto Veneto, Venice, Italy

\footnotetext{
* Speaker.
} 


\section{Introduction}

After the discovery of neutrino oscillations and the precise measurement of the mass squared differences and of the mixing angles in the past 20 years, the determination of leptonic $\mathrm{CP}$ violation (CPV) is one of the most compelling question in particle physics. In the standard three-neutrino mixing scheme, one or three $\mathrm{CP}$ violating phases are present in the Pontecorvo-Maki-NagakawaSakata unitary mixing matrix, $U_{\mathrm{PMNS}}$, [1]: one phase $\delta$ if neutrinos are Dirac particles, and three if they are Majorana ones. $\delta$ enters in neutrino oscillations, in particular in the appearance probability in long-baseline neutrino oscillation experiments. Recently, hints in favour of CP violation have been found when one combines the data from the long baseline oscillation experiment T2K (and NOvA) with that from reactor experiments, in particular Daya Bay. In fact, the former reports a rather large neutrino appearance oscillation probability which depends on $\theta_{13}, \theta_{23}$, the mass ordering and crucially $\delta$, while the latter constrains very strongly $\theta_{13}$ alone. The most recent combined analysis in Ref. [2] gives a best fit value of $-54^{\circ}\left(+30^{\circ},-69^{\circ}\right)\left(-106^{\circ}\left( \pm 63^{\circ}\right)\right)$ for normal ordering (inverted ordering), while at $3 \sigma$ all values, including $\mathrm{CP}$ conserving ones, are allowed. Current superbeams, i.e. T2K and NOvA, and future long-baseline experiments, e.g. DUNE, T2HK, will allow to test these first hints. If neutrinos are Majorana particles, two additional phases cannot be rotated away and are physical. They enter in lepton number violating processes, the most sensitive of which is neutrinoless double beta decay. In principle, a precise measurement of the effective Majorana mass, combined with an accurate determination of neutrino masses, e.g. from cosmological observations, would allow to test if Majorana CP violation is present. However, this requires not only very sensitive experiments but also a precise computation of the nuclear matrix elements for neutrinoless double beta decay, which is beyond the current state of the art, see e.g. [3].

$\mathrm{CP}$ violation, together with the violation of lepton number, can play a crucial role in the generation of the baryon asymmetry of the Universe via the leptogenesis mechanism. In this article we will review the possible connection between the measurable leptonic CP violation at low energy, and in particular $\delta$, and leptogenesis, focussing on see-saw type I models with hierarchical right handed neutrinos.

\section{CP violation and the baryon asymmetry}

The origin of the matter-antimatter asymmetry in the Universe is one of the most compellings questions in cosmology. Its value has been well measured using the cosmic microwave background $(\mathrm{CMB})$ radiation by Planck [4]

$$
Y_{B}^{\mathrm{CMB}} \simeq(8.67 \pm 0.09) \times 10^{-10},
$$

where $Y_{B}$ is the baryon to photon ratio at recombination. An independent measurement is given by Big Bang Nucleosynthesis $(\mathrm{BBN}), Y_{B}^{\mathrm{BBN}} \simeq(8.10 \pm 0.85) \times 10^{-10}$, in good agreement with the CMB one [5].

Assuming that the Universe had no baryon asymmetry to start with, as suggested by inflationary models, the baryon asymmetry can be dynamically generated if the conditions suggested by A. D. Sakharov in 1967 are satisfied [6]:

- Baryon number (or Lepton number, for the leptogenesis mechanism) violation. 
- $\mathrm{C}$ and $\mathrm{CP}$ violation. If $\mathrm{CP}$ is conserved, every reaction which produces a particle will be accompanied by an opposite one, with no creation of a net baryon number.

- Departure from thermal equilibrium. This condition is readily satisfied in the Early Universe by its expansion.

Several mechanisms have been proposed as the origin of the baryon asymmetry. A very popular and successfull explanation is leptogenesis [7]. It relies on the fact that, as $B-L$ is conserved both at the perturbative and non-perturbative level, a net $B-L$, (e.g., a lepton number) would induce a net baryon number via sphaleron effects [8].

\subsection{The see-saw mechanism and leptogenesis}

Leptogenesis is particularly appealing because it naturally occurs in see-saw models [9], which can explain neutrino masses and their smallness. Here, for conciseness we focus on see-saw type I models. Heavy right-handed (RH) Majorana neutrinos, $N_{i}$, singlets under the SM gauge symmetry group, are introduced in the theory and the resulting Lagrangian contains a Yukawa term which couples $N_{i}$ with the Higgs and the leptonic doublet. Once the Higgs gets a vacuum expectation value, a Dirac mass terms arises and the neutrinos mass terms in the Lagrangian read

$$
-\mathscr{L}_{\text {mass }}=\bar{N}_{i}\left(m_{D}\right)_{i j} v_{L j}+\text { h.c. }+\frac{1}{2} \overline{\left(N_{i}\right)^{c}}\left(M_{R}\right)_{i j} N_{j},
$$

where $m_{D}$ and $M_{R}$ are the Dirac and Majorana mass matrices, respectively. For $M_{R} \gg m_{D}$, light neutrino masses are induced according to the see-saw [9] formula

$$
m_{v}=U_{\mathrm{PMNS}}^{*} D_{m} U_{\mathrm{PMNS}}^{\dagger} \simeq m_{D}^{T} M_{R}^{-1} m_{D} .
$$

Here, $D_{m}$ is a diagonal matrix containing the three light masses $m_{1,2,3}$.

The see-saw models can satisfy the Sakharov conditions as i) lepton number is violated by the Majorana $M_{R}$ term; ii) CP violation can be present in the $m_{D}$ matrix if some of its elements are complex; iii) the departure from equilibrium is due to the $N_{i}$ decays in the expanding Universe, once the temperature drops below their mass.

\subsubsection{The one-flavor approximation}

At high temperatures, $T>10^{12} \mathrm{GeV}$, different leptonic flavors cannot be distinguished as their Yukawa interactions are out of equilibrium. In this case, assuming $M_{1} \ll M_{2} \ll M_{3}$, only one CP-asymmetry needs to be considered $[7,10,11]$ :

$$
\varepsilon_{1}=\frac{\Gamma\left(N_{1} \rightarrow \Phi^{-} \ell^{+}\right)-\Gamma\left(N_{1} \rightarrow \Phi^{+} \ell^{-}\right)}{\Gamma\left(N_{1} \rightarrow \Phi^{-} \ell^{+}\right)+\Gamma\left(N_{1} \rightarrow \Phi^{+} \ell^{-}\right)} \simeq \frac{3}{16 \pi v^{2}} \sum_{j \neq 1} \frac{\operatorname{Im}\left(m_{D} m_{D}^{\dagger}\right)_{1 j}^{2}}{\left(m_{D} m_{D}^{\dagger}\right)_{11}} \frac{M_{1}}{M_{j}},
$$

where $\Phi$ and $\ell$ are the Higgs field and the charged leptons, respectively. $v$ is the electroweak symmetry breaking scale. This CP-asymmetry is partially washed-out by inverse decays and other lepton number violating processes, due to the fact that the lepton asymmetry production in $N_{1}$ decays is not instantaneous. These "wash-out" effects are flavor-independent and can be parameterized by $\widetilde{m_{1}} \propto\left(m_{D} m_{D}^{\dagger}\right)_{11}$, proportional to the total decay rate of $N_{1}$. Finally, the lepton asymmetry is 
converted into a baryon one by sphaleron effects, $Y_{B}=c_{s} Y_{B-L}$, where $c_{s}$ is a coefficient typically of order $0.2-0.4$, depending on the specific model.

The observed baryon asymmetry is then given by

$$
Y_{B} \simeq-c_{s} \frac{\varepsilon_{1}}{g_{*}} \eta\left(\widetilde{m_{1}}\right),
$$

where $\eta\left(\widetilde{m_{1}}\right)$ accounts for the washing out due to inverse decays.

It should be noted that the resulting baryon asymmetry depends on the trace of the $\mathrm{CP}$ asymmetries over flavours, $\varepsilon_{1}$, times a function of the trace over flavours of the decay rate of $N_{1}$.

\subsubsection{Flavor effects}

Once the interactions due to the charged lepton Yukawa couplings get into equilibrium, at $T \ll$ $10^{12} \mathrm{GeV}$ for $\tau$ leptons and at $T \sim 10^{9} \mathrm{GeV}$ for muons, different flavours become distinguishable and the asymmetry and wash-out effects become flavour-dependent. The total baryon asymmetry is obtained summing three contributions $[12,13,14,15]$ : the CP asymmetry in each lepton flavor $l$, washed-out by the same-lepton number violating processes. The asymmetry in each flavor $l$ is given by

$$
\varepsilon_{l}=\frac{3}{16 \pi v^{2}} \frac{1}{\left(m_{D} m_{D}^{\dagger}\right)_{11}} \operatorname{Im}\left(\sum_{j}\left(\left(m_{D}\right)_{1 l}\left(m_{D} m_{D}^{\dagger}\right)_{1 j}\left(m_{D}^{*}\right)_{j l}\right)\right) \frac{M_{1}}{M_{j}} .
$$

Similarly, one has to consider the "wash-out mass parameter" for each flavour $l[13,15], \widetilde{m_{l}} \propto$ $\left|\left(m_{D}\right)_{1 l}\right|^{2}$, which depends on the decay rate of $N_{1}$ to the leptons of flavour $l$.

As an example, here we will consider temperatures $\left(10^{9} \leq T \sim M_{1} \ll 10^{12}\right) \mathrm{GeV}$, for which only the interactions mediated by the $\tau$ Yukawa coupling are in equilibrium. The final baryon asymmetry is well approximated by [15]

$$
Y_{B} \simeq-\frac{12}{37 g_{*}}\left(\varepsilon_{2} \eta\left(\frac{417}{589} \widetilde{m_{2}}\right)+\varepsilon_{\tau} \eta\left(\frac{390}{589} \widetilde{m_{\tau}}\right)\right),
$$

where $\varepsilon_{2}=\varepsilon_{e}+\varepsilon_{\mu}, \widetilde{m_{2}}=\widetilde{m_{e}}+\widetilde{m_{\mu}}$. It should be noted that, differently from the "one-flavor approximation", the total baryon number is not proportional to $\varepsilon_{1}$.

\section{Testing the ingredients for leptogenesis: leptonic CP violation}

The appearance channel $v_{\mu} \rightarrow v_{e}$ in long-baseline neutrino oscillation experiments depends on the $\delta$ phase and offers the opportunity to hunt for leptonic CP violation. The probability can be approximated, expanding to second order in the small parameters $\sin \theta_{13}$ and $\Delta m_{21}^{2} / \Delta m_{31}^{2}$, as [16]:

$$
\begin{aligned}
P_{v_{\mu} \rightarrow v_{e}} \approx & 4 \sin ^{2} \theta_{13} \sin ^{2} \theta_{23} \frac{\sin ^{2} \Delta(1-A)}{(1-A)^{2}}+\alpha^{2} \sin ^{2} 2 \theta_{12} \cos ^{2} \theta_{23} \frac{\sin ^{2} A \Delta}{A^{2}} \\
& +2 \alpha \sin \theta_{13} \sin 2 \theta_{12} \sin 2 \theta_{23} \cos (\Delta \pm \delta) \frac{\sin \Delta A}{A} \frac{\sin \Delta(1-A)}{1-A}
\end{aligned}
$$

where the following definitions hold

$$
\alpha \equiv \Delta m_{21}^{2} / \Delta m_{31}^{2}, \quad \Delta \equiv \frac{\Delta m_{31}^{2} L}{4 E}, \quad A \equiv \frac{2 E V}{\Delta m_{31}^{2}} .
$$


Here, $L$ is the baseline, $E$ is the neutrino energy, and $V$ is the effective matter potential [17], taken to be constant over these baselines. The plus (minus) sign holds for neutrinos (antineutrinos), and for antineutrinos $V$ needs to be changed to $-V$, i.e. $A \rightarrow-A$.

The first term in the probability is being referred to as "atmospheric term" given the dependence on $\Delta m_{31}^{2}$ and is dominant due to the observed large value of $\theta_{13}, \sin ^{2} \theta_{13} \approx 0.022$. This term provides the strongest sensitivity to matter effects and allows to determine the neutrino mass ordering with the present generation and future long-baseline oscillation experiments, such as NOvA, DUNE, and a neutrino factory. The second term is typically very small as it is suppressed by two powers of $\alpha$. The third term, usually called the "CP violating" term, depends on the $\delta$ phase and is suppressed due to $\Delta m_{21}^{2}$, although it depends only linearly on $\sin \theta_{13}$. This implies that the determination of $\mathrm{CP}$ violation is challenging as it requires the measurement of the probability at the few percent level.

Moreover, the extraction of the unknown parameters, i.e. the sign of $\Delta m_{31}^{2}, \delta$, the octant of $\theta_{23}$, is complicated by the fact that they enter the probability in a complex manner. This leads to the well known problem of degeneracies [18], so that, even if the probabilities were measured to a high precision, there would remain uncertainties in the reconstruction of the unknown parameters. A particularly relevant degeneracy, highlighted in [19], is the one between $\theta_{23}-\delta-\theta_{13}$, for which the synergy between the appearance and disappearance channels can have a relevant role. Generically, having information at different energies, combining oscillation channels and using inputs from other experiments, such as reactor or atmospheric neutrino ones, helps solving this problem and improve significantly the reach of long baseline experiments.

Experimentally long baseline neutrino oscillation experiments exploit beams sourced in pion and muon decays and large detectors. Specifically in superbeams, the muon neutrino (and antineutrino) beam is produced at an accelerator complex in which high energy protons are impinged on a target. The collisions produce large quantities of pions which are focussed and decay sourcing to a collimated beam of muon neutrinos. The latter travels and can interact in large detectors, which have the capabilities to reconstruct $v_{e}$ and $v_{\mu}$. Typical choices are Water Cherenkov, for $E_{v}<1 \mathrm{GeV}$, Liquid Argon (LAr) or scintillator (LSc) ones. Important backgrounds to the appearance channel are: i) mis-identified $\pi^{0}$ produced in neutral current interactions, as one of the $\gamma_{S}$ from the pion decay $\pi^{0} \rightarrow \gamma \gamma$ is not reconstructed; ii) intrinsic $v_{e}$ from the beam, usually coming from kaon decays, at a level of $0.5 \%-1 \%$; iii) mis-reconstructed muon neutrinos. Systematic errors, both on the signal and on the backgrounds, due to the beam and the detector, such as e.g. the cross sections, overall normalisation, are crucial to establish the sensitivity of the setup. In this, the near detector will play an important role.

Two long-baseline neutrino experiments, $\mathrm{T} 2 \mathrm{~K}$ and NOvA, are currently taking data. Thanks to the large value of $\theta_{13}$ they will be able to provide some information on the mass ordering and on $\mathrm{CP}$ violation, when combined with a precise measurement of $\theta_{13}$ from reactor neutrino experiments.

T2K [20]. The beam from the J-PARC accelerator complex has a nominal power of 750 $\mathrm{kW}$ and has recently reached $350 \mathrm{~kW}$. The detector is the $22.5 \mathrm{kton}$ Super-Kamiokande Water Cerenkov detector located at a distance of $295 \mathrm{~km}$ and at an off-axis angle of $2.5^{\circ}$. A combination of the neutrino and antineutrino runs, together with a precise value of $\theta_{13}$ will allow to reach a $2 \sigma$ sensitivity for maximal CPV in the favorable region $\delta<0$, see e.g. Refs. [21]. Interestingly, in July 2015 T2K reported the first results for its antineutrino run. 
NOvA [22]. This experiment uses the NuMI beam with $700 \mathrm{~kW}$ and a 14 kton totally active plastic scintillator detector located $810 \mathrm{~km}$ away off-axis. NOvA presented the first results from the neutrino run in August 2015. It has been shown that NOvA has a similar sensitivity to CPV as $\mathrm{T} 2 \mathrm{~K}$, at a 1.5-2 $\sigma$ for a broader range of values [23], and that the combination of these two experiments could even reach a $3 \sigma$ result for particularly favourable values of $\delta$.

The prospective sensitivity significantly depends on the value of $\theta_{23}$ worsening going from lower to higher values of $\theta_{23}$. For these experiments, the sensitivity is statistics-limited and additional years of running would lead to significant improvements.

DUNE [24, 25]. The DUNE experiment, exploiting the LBNF facility at Fermilab, is a proposal in the US which uses a beam sourced at the Main Injector at Fermilab with 1.2 MW of power. Differently from T2K and NOvA, this experiment would have the advantage of a broad energy spectrum and consequently the ability to get information on the probability at different energies. The chosen detector comprises 410 kton modules of LAr TPC, located at the Sanford Underground Research Facility site, at a distance of $1300 \mathrm{~km}$ from Fermilab. A recent optimisation of the beam has allowed to improve significantly the reach in CP violation: DUNE will achieve a sensitivity $>3 \sigma$ for $75 \%$ of the values of $\delta$ in 1320 kton MW years and reach a $5 \sigma$ discovery for $50 \%$ of the values in 810 kton MW years (for the most recent analysis see DUNE CDR document in Ref. [25]).

J-PARC to Hyper-Kamiokande long-baseline experiment (T2HK) [26, 27]. This facility uses a beam sourced at the JPARC accelerator and aimed at a 0.99 Mton Water Cherenkov detector located $2.5^{\circ}$ off-axis at $295 \mathrm{~km}$ in the Kamiokande mine. Considering a total exposure of $7.5 \mathrm{MW}$ times $10^{7}$ seconds, leptonic CP violation can be established at $3 \sigma$ for $76 \%$ of the values of $\delta$ and discovered at $5 \sigma$ for $58 \%$ of them. The advantages of this proposal are the excellent energy resolution for quasi-elastic interactions at these energies, the large number of events and the low intrinsic background.

$\mathbf{E S S} v \mathbf{S B}$ [28]. Differently from other options, this $10 \mathrm{MW}$ beam, sourced at the European Spallation Source, is peak around the second oscillation maximum in order to maximise the sensitivity to CP violation. It is aimed at a 500 kton Water Cherenkov detector which could be located at a distance between 300 and $550 \mathrm{~km}$. This setup has the ability to discover CP violation at $5 \sigma$ for up to $50 \%$ of the values of $\delta$. Additional studies are currently ongoing in order to further optimize this facility.

Neutrino factory [29, 30, 31, 32, 33]. Neutrinos are produced by high energy muons which decay producing a collimated beam of muon neutrinos and electron antineutrinos. A magnetised detector, a 100 kton iron MIND, is needed in order to distinguish the muon produced by interacting $v_{\mu}$ from $v_{\mu} \rightarrow v_{\mu}$ oscillations, from the antimuons arising from the interactions of $\bar{v}_{\mu}$ from the appearance channel. The baseline configuration uses $10 \mathrm{GeV}$ muons and $2000 \mathrm{~km}$. Thanks to the high number of events, very low backgrounds and the wide and well known energy spectrum, this setup achieves a superior performance with $\mathrm{CP}$ violation which could be discovered for over $70 \%$ of the values of $\delta$.

Other type of setups. For the sake of completeness, we note that there are non-long-baseline strategies to search for leptonic CP violation. DAE $\delta$ ALUS (Decay-At-rest Experiment for $\delta \mathrm{CP}$ studies At the Laboratory for Underground Science) [34] uses a cyclotron-driven muon antineutrino beam and a very large detector optimised for low energies, such as a 50 kton liquid scintillator 
one, e.g. LENA, or a megaton scale Water Cherenkov one, e.g. a gadolinium-doped 300 kton or Hyper-K detectors. Placing the source at different distances, e.g. $1.5 \mathrm{~km}, 8 \mathrm{~km}$ and $20 \mathrm{~km}$, $\mathrm{CP}$ violation can be searched for. Finally, very large detectors for atmospheric neutrinos, e.g. SuperPINGU, can provide information on $\mathrm{CP}$ violation using subGeV energy neutrinos. Depending on the specific detector performance achievable, maximal $\mathrm{CP}$ violation could be distinguished from the CP conserving value $\delta=0$ with a significance between $3 \sigma$ and $8 \sigma$, see Ref. [35].

When comparing the sensitivities of different setups, a word of caution is in order: the precise physics reach of each configuration is strongly dependent on the assumption made on the beam, detector, oscillation parameters and systematic errors.

\section{The connection between low-energy leptonic CPV and the baryon asymmetry}

Establishing a connection between the parameters at low energy (in particular, the CP violating phases and the presence of lepton number violation), and at high energy (relevant in leptogenesis) is very interesting. Here, we focus the discussion on see-saw type I models with 3 hierarchical $N_{i}$ masses. We ask the following question: could the CP violating phase $\delta$ be responsible for the baryon asymmetry?

In a generic model, the number of parameters in the see-saw Lagrangian is larger than that of the measurable ones: for three heavy neutrinos, at high energy the theory contains 3 heavy masses, 9 real parameters and 6 phases in the Dirac mass matrix, while at low energy only 9 are in principle accessible - 3 angles, 3 masses and 3 phases (of these only one Dirac and potentially one Majorana phase could ever be measured). This implies that a "one-to-one connection" between the low-energy and the high energy parameters, specifically the phases, cannot be established in a model independent way. However, typically, models implementing symmetries, textures or other tools to explain the low energy flavour structure, have a reduced number of parameters and might allow such a connection.

In the basis in which both $M_{R}$ and the charged lepton mass matrix are real and diagonal, the orthogonal parametrization for the Dirac mass [36] turns out to be very useful, as it allows to separate measurable parameters from high energy unknown ones:

$$
m_{D} \simeq \sqrt{M_{R}} \mathbf{R} D_{m}^{1 / 2} U_{\mathrm{PMNS}}^{\dagger},
$$

where $\mathbf{R}$ is a complex orthogonal matrix, which contains 3 unknown real parameters and 3 unkown phases.

Using this parameterisation, in the "one-flavor" approximation, the CP asymmetry can be rewritten as:

$$
\varepsilon_{1}=-\frac{3 M_{1}}{16 \pi v^{2}} \frac{\operatorname{Im}\left(\sum_{\alpha \beta \rho} m_{\rho}^{1 / 2} m_{\beta}^{3 / 2} U_{\alpha \rho}^{*} U_{\alpha \beta} R_{1 \beta} R_{1 \rho}\right)}{\sum_{\beta} m_{\beta}\left|R_{1 \beta}\right|^{2}}=-\frac{3 M_{1}}{16 \pi v^{2}} \frac{\operatorname{Im}\left(\sum_{\rho} m_{\rho}^{2} R_{1 \rho}^{2}\right)}{\sum_{\beta} m_{\beta}\left|R_{1 \beta}\right|^{2}} .
$$

It is apparent that the unitary mixing matrix $U_{\mathrm{PMNS}}$ does not enter directly into the expression for the lepton asymmetry. Therefore, in general an observation of CP violation at low energy would not provide any information on the generation of a baryon asymmetry in the Early Universe. 
This conclusion does not hold if leptogenesis takes place at $T \ll 10^{12} \mathrm{GeV}$. In this case flavors are distinguishable and each flavor asymmetry must be considered separately:

$$
\varepsilon_{\alpha}=-\frac{3 M_{1}}{16 \pi v^{2}} \frac{\operatorname{Im}\left(\sum_{\beta \rho} m_{\beta}^{1 / 2} m_{\rho}^{3 / 2} U_{\alpha \beta}^{*} U_{\alpha \rho} R_{1 \beta} R_{1 \rho}\right)}{\sum_{\beta} m_{\beta}\left|R_{1 \beta}\right|^{2}} .
$$

Since each flavour CP asymmetry is weighted by the corresponding wash out parameter, the total lepton number depends directly on the CP violating phases at low energy [37, 38, 14, 15].

Asking if the $\delta$ phase can be responsible for the baryon asymmetry can be analysed by imposing that the matrix $R$ is real and setting the Majorana phases to CP-conserving values [37, 38]. In this case, the flavor CP asymmetries $\varepsilon_{2}=-\varepsilon_{\tau}$ and, consequently, the baryon asymmetry depend exclusively on the low energy phases, specifically $\delta$. It is now necessary to recast the baryon asymmetry in terms of $\delta$ and to check that the obtained values can be compatible with the observations. For concreteness, we consider the normal hierarchical mass spectrum for light neutrinos, $m_{1} \ll m_{2} \ll m_{3}$. We take the simplest case of decoupling of the heaviest right-handed neutrino, $R_{11}=0$. Detailed results for generic $R$ and for other choices of neutrino mass spectrum can be found in Ref. [38]. The baryon asymmetry is given by [38]:

$$
\left|Y_{B}\right| \cong 2.4 \times 10^{-11}|\sin \delta|\left(\frac{s_{13}}{0.15}\right)\left(\frac{M_{1}}{10^{11} \mathrm{GeV}}\right)
$$

Here, we have taken $R_{12}=0.92$ and $R_{13}=0.39$ which approximately maximize the CP asymmetry. We have used the current best fit values for the oscillation parameters. In order to generate the observed baryon asymmetry, $\left|Y_{B}\right| \simeq 8.59 \times 10^{-10}$, for maximal CP violation, $|\sin \delta| \sim 1$, in the case analised, we should have $M_{1} \sim 3.5 \times 10^{11} \mathrm{GeV}$ [38]. Recalling that flavour effects in leptogenesis are developed for $M_{1} \leq 5 \times 10^{11} \mathrm{GeV}$, this implies that $\mathrm{CP}$ needs to be violated maximally, or nearly maximally. These values are in agreement with current hints and will be at reach of future long baseline neutrino oscillation experiments.

\section{Conclusions}

The observation of lepton number non-conservation and of leptonic CP violation in long baseline neutrino oscillations, at reach in current and future experiments, would have important implications for our understanding of the origin of the baryon asymmetry. They would constitute a strong indication (even if not a proof) in favour of leptogenesis as the mechanism of generation of the baryon asymmetry of the Universe.

\section{Acknowledgments}

The author would like to thank the organisers for the excellent conference. Part of this work has been supported by the European Research Council under ERC Grant "NuMass" (FP7-IDEAS-ERC ERC-CG 617143) and by the European Commission FP7 ITN Invisibles (Marie Curie Actions, PITN-GA-2011-289442). 


\section{References}

[1] B. Pontecorvo, Zh. Eksp. Teor. Fiz. 33, 549 (1957) and 34, 247 (1958); Z. Maki, M. Nakagawa and S. Sakata, Prog. Theor. Phys. 28, 870 (1962).

[2] M. C. Gonzalez-Garcia, M. Maltoni and T. Schwetz, JHEP 1411, 052 (2014) [arXiv:1409.5439 [hep-ph]].

[3] S. M. Bilenky, C. Giunti, C. W. Kim and S. T. Petcov, Phys. Rev. D 54, 4432 (1996) [hep-ph/9604364]; S. Pascoli, S. T. Petcov and L. Wolfenstein, Phys. Lett. B 524, 319 (2002) [hep-ph/0110287]; S. Pascoli, S. T. Petcov and T. Schwetz, Nucl. Phys. B 734, 24 (2006) [hep-ph/0505226]; H. Minakata, H. Nunokawa and A. A. Quiroga, arXiv:1402.6014 [hep-ph]; S. Dell'Oro, S. Marcocci and F. Vissani, Phys. Rev. D 90 (2014) 3, 033005 [arXiv:1404.2616 [hep-ph]].

[4] P. A. R. Ade et al. [Planck Collaboration], Astron. Astrophys. 571, A16 (2014) [arXiv:1303.5076 [astro-ph.CO]]; P. A. R. Ade et al. [Planck Collaboration], arXiv:1502.01589 [astro-ph.CO].

[5] F. Iocco, G. Mangano, G. Miele, O. Pisanti and P. D. Serpico, Phys. Rept. 472, 1 (2009) [arXiv:0809.0631 [astro-ph]].

[6] A. D. Sakharov, JTEP Lett. 6, 24 (1967).

[7] M. Fukugita and T. Yanagida, Phys. Lett. B 174, 45 (1986).

[8] J.A. Harvey, M.S. Turner, Phys. Rev. D 42, 3344 (1990.)

[9] P. Minkowski, Phys. Lett. B 67, 421 (1977); M. Gell-Mann, P. Ramond, and R. Slansky in Supergravity, p. 315, edited by F. Nieuwenhuizen and D. Friedman, North Holland, Amsterdam, 1979; T. Yanagida, Proc. of the Workshop on Unified Theories and the Baryon Number of the Universe, edited by O. Sawada and A. Sugamoto, KEK, Japan 1979; R.N. Mohapatra, G. Senjanovic, Phys. Rev. Lett. 44, 912 (1980).

[10] M. A. Luty, Phys. Rev. D 45, 455 (1992); M. Flanz, E. A. Paschos and U. Sarkar, Phys. Lett. B 345, 248 (1995) [Erratum-ibid. B 382, 447 (1996)] [hep-ph/9411366]; M. Plumacher, Z. Phys. C 74, 549 (1997) [hep-ph/9604229].

[11] L. Covi, E. Roulet and F. Vissani, Phys. Lett. B 384, 169 (1996) [hep-ph/9605319]; M. Flanz, E. A. Paschos, U. Sarkar and J. Weiss, Phys. Lett. B 389, 693 (1996) [hep-ph/9607310]; A. Pilaftsis, Phys. Rev. D 56, 5431 (1997) [hep-ph/9707235]; W. Buchmuller and M. Plumacher, Phys. Lett. B 431, 354 (1998) [hep-ph/9710460].

[12] R. Barbieri, P. Creminelli, A. Strumia and N. Tetradis, Nucl. Phys. B 575, 61 (2000) [hep-ph/9911315].

[13] A. Abada, S. Davidson, F. X. Josse-Michaux, M. Losada and A. Riotto, JCAP 0604, 004 (2006) [hep-ph/0601083].

[14] E. Nardi, Y. Nir, E. Roulet and J. Racker, JHEP 0601, 164 (2006) [hep-ph/0601084].

[15] A. Abada, S. Davidson, A. Ibarra, F.-X. Josse-Michaux, M. Losada and A. Riotto, JHEP 0609, 010 (2006) [hep-ph/0605281].

[16] A. Cervera, A. Donini, M. B. Gavela, J. J. Gomez Cadenas, P. Hernandez, O. Mena and S. Rigolin, Nucl. Phys. B 579, 17 (2000) [Erratum-ibid. B 593, 731 (2001)] [hep-ph/0002108]; M. Freund, Phys. Rev. D 64, 053003 (2001) [hep-ph/0103300]; E. K. Akhmedov, R. Johansson, M. Lindner, T. Ohlsson and T. Schwetz, JHEP 0404, 078 (2004) [hep-ph/0402175]; K. Asano and H. Minakata, JHEP 1106, 
022 (2011) [arXiv:1103.4387 [hep-ph]]; S. K. Agarwalla, Y. Kao and T. Takeuchi, JHEP 1404, 047 (2014) [arXiv:1302.6773 [hep-ph]]; H. Minakata and S. J. Parke, arXiv:1505.01826 [hep-ph].

[17] L. Wolfenstein, Phys. Rev. D 17, 2369 (1978); S. P. Mikheev and A. Y. Smirnov, Sov. J. Nucl. Phys. 42, 913 (1985) [Yad. Fiz. 42, 1441 (1985)].

[18] G. L. Fogli and E. Lisi, Phys. Rev. D 54, 3667 (1996) [hep-ph/9604415]; J. Burguet-Castell, M. B. Gavela, J. J. Gomez-Cadenas, P. Hernandez and O. Mena, Nucl. Phys. B 608, 301 (2001) [hep-ph/0103258]; H. Minakata and H. Nunokawa, JHEP 0110, 001 (2001) [hep-ph/0108085]; V. Barger, D. Marfatia and K. Whisnant, Phys. Rev. D 65, 073023 (2002) [hep-ph/0112119]; H. Minakata, H. Nunokawa and S. J. Parke, Phys. Rev. D 66, 093012 (2002) [hep-ph/0208163]; Phys. Rev. D 70, 093011 (2004) [hep-ph/0408070].

[19] P. Coloma, H. Minakata and S. J. Parke, Phys. Rev. D 90 (2014) 093003 [arXiv:1406.2551 [hep-ph]].

[20] K. Abe et al. [T2K Collaboration], Phys. Rev. Lett. 107, 041801 (2011) [arXiv:1106.2822 [hep-ex]]; K. Abe et al. [T2K Collaboration], Phys. Rev. D 91 (2015) 7, 072010 [arXiv:1502.01550 [hep-ex]].

[21] P. Huber, M. Lindner, T. Schwetz and W. Winter, JHEP 0911, 044 (2009) [arXiv:0907.1896 [hep-ph]]; M. Ghosh, P. Ghoshal, S. Goswami and S. K. Raut, Nucl. Phys. B 884, 274 (2014) [arXiv:1401.7243 [hep-ph]]; P. A. N. Machado, H. Minakata, H. Nunokawa and R. Zukanovich Funchal, JHEP 1405, 109 (2014) [arXiv:1307.3248].

[22] D. S. Ayres et al. [NOvA Collaboration], hep-ex/0503053.

[23] M. Blennow, P. Coloma and E. Fernandez-Martinez, JHEP 1503 (2015) 005 [arXiv:1407.3274 [hep-ph]].

[24] C. Adams et al. [LBNE Collaboration], arXiv:1307.7335 [hep-ex]

[25] For the DUNE CDR, see http://docs.dunescience.org/

[26] K. Abe, T. Abe, H. Aihara, Y. Fukuda, Y. Hayato, K. Huang, A. K. Ichikawa and M. Ikeda et al., arXiv:1109.3262 [hep-ex].

[27] K. Abe et al. [Hyper-Kamiokande Proto- Collaboration], PTEP 2015 (2015) 5, $053 \mathrm{C} 02$ [arXiv:1502.05199 [hep-ex]].

[28] E. Baussan et al. [ESSnuSB Collaboration], arXiv:1309.7022 [hep-ex].

[29] S. Geer, Phys. Rev. D 57, 6989 (1998) [Erratum-ibid. D 59, 039903 (1999)] [hep-ph/9712290].

[30] A. De Rujula, M. B. Gavela and P. Hernandez, Nucl. Phys. B 547, 21 (1999) [hep-ph/9811390].

[31] S. Geer, O. Mena and S. Pascoli, Phys. Rev. D 75, 093001 (2007) [hep-ph/0701258 [HEP-PH]].

[32] A. Bandyopadhyay et al. [ISS Physics Working Group Collaboration], Rept. Prog. Phys. 72, 106201 (2009) [arXiv:0710.4947 [hep-ph]].

[33] A. D. Bross, M. Ellis, S. Geer, O. Mena and S. Pascoli, Phys. Rev. D 77, 093012 (2008) [arXiv:0709.3889 [hep-ph]].

[34] C. Aberle, A. Adelmann, J. Alonso, W. A. Barletta, R. Barlow, L. Bartoszek, A. Bungau and A. Calanna et al., arXiv:1307.2949.

[35] S. Razzaque and A. Y. Smirnov, JHEP 1505 (2015) 139 [arXiv:1406.1407 [hep-ph]].

[36] J. A. Casas and A. Ibarra, Nucl. Phys. B 618, 171 (2001) [hep-ph/0103065].

[37] S. Pascoli, S. T. Petcov and A. Riotto, Phys. Rev. D 75, 083511 (2007) [hep-ph/0609125].

[38] S. Pascoli, S. T. Petcov and A. Riotto, Nucl. Phys. B 774, 1 (2007) [hep-ph/0611338]. 